\title{
Gastric Neuroendocrine Carcinoma in a Dog
}

\author{
Nadja Herbach ${ }^{1}$, Stefan Unterer ${ }^{2}$, Walter Hermanns ${ }^{1}$ \\ ${ }^{1}$ Institute of Veterinary Pathology, Centre for Clinical Veterinary Medicine, Ludwig Maximilians University Munich, Munich, Ger- \\ many; ${ }^{2}$ Clinic of Small Animal Medicine, Centre for Clinical Veterinary Medicine, Ludwig Maximilians University Munich, Munich, \\ Germany. \\ Email: n.herbach@lmu.de
}

Received July $11^{\text {th }}, 2012$; revised August $24^{\text {th }}, 2012$; accepted September $3^{\text {rd }}, 2012$

\begin{abstract}
A gastric biopsy specimen from a 14-year-old Yorkshire terrier was analysed using light microscopy, immunohistochemistry and transmission electron microscopy. The biopsies were obtained from a $3 \times 3 \times 4 \mathrm{~cm}$ mass located at the gastric cardia. Light microscopy revealed solid nests and sheets of tumour cells separated by abundant fibrovascular stroma in all gastric layers. The tumour cells were monomorphic, with eosinophilic cytoplasm and demonstrated palisading at the periphery. Nuclei were round to oval with densely packed chromatin and inconspicuous nucleoli. The tumour cells immunohistochemically stained strongly positive for chromogranin A, synaptophysin, and pan-cytokeratin. Electron microscopy revealed electron dense neurosecretory granules of $100-150 \mathrm{~nm}$ in diameter. Metastases were found in regional lymph nodes. Gastric neuroendocrine carcinoma was diagnosed, according to the histological, immunohistochemical and electron microscopic features.
\end{abstract}

Keywords: Gastric Carcinoid, Gastric Neuroendocrine Carcinoma; Dog; Stomach

\section{Introduction}

Neuroendocrine (NE) cells may form glands (adrenal medulla, parathyroid, paraganglia, adenohypophysis) or may be diffusely distributed throughout the body in different organs, i.e. the gastrointestinal tract, pancreas, skin, thymus, thyroid, lung, bilary and urinary tract. These diffusely distributed NE cells belong to the disseminated/diffuse neuroendocrine system, DNES [1]. The DNES produces and secretes peptides and amines that are essential for regulation of carbohydrate, protein and fat metabolism [2]. NE cells can be identified, using immunohistochemistry for synaptophysin, chromogranin A, neural cell adhesion molecule (NCAM, CD56) and neuron specific enolase (NSE) [1].

Tumours arising from the DNES, i.e. neuroendocrine tumours (NET) were formerly called argentaffinoma, enterochromaffin cell tumour, carcinoid or apudoma. NET of the gastrointestinal tract of domestic animals are rare, with few case reports of carcinoids occurring in the intestine of adult dogs, cats, cows and a horse [3]. Neuroendocrine neoplasms (NEN) of the pancreas and gastrointestinal tract in man are considered potentially malignnant and are classified as well differentiated NEN (NET with low grade malignant behaviour G1 (carcinoid) or uncertain behaviour G2), or as poorly differentiated NEN or neuroendocrine carcinoma (NEC) with highgrade malignant behaviour (G3), exhibiting a high proliferation rate. NEC can be further subdivided into a small-cell and a large-cell variant [1,4]. In animals, intestinal carcinoids are also considered malignant, grow locally invasive, infiltrating the submucosa and muscularis, and metastasize widely [3].

In humans, gastric NETs are the most frequent NETs and can be divided into four subtypes. Type 1 is the most frequent gastric NET, arising secondary to autoimmune chronic atrophic corpus gastritis where loss of gastric parietal cells leads to insufficient production of intrinsic factor and hypochloric acid. Achlorhydria stimulates gastrin-producing G-cells, causing hypergastrinaemia, which in turn stimulates proliferation of enterochromaffin-like (ECL) cells, and ultimately leads to the formation of multiple, usually small tumours [1]. The other types of gastric NETs are rare and include type 2 NETs that occur in association with multiple endocrine neoplasia (MEN1) and Zollinger-Ellison syndrome. Type 3 are sporadic NETs that are solitary, and Type 4 NETs are undifferentiated gastric carcinomas that are usually large and have metastasized at the time of diagnosis [1,5]. In animals, no association between gastrinoma and gastric NET can be made due to scarcity of both forms of NETs [2].

\section{Methods}

Biopsy samples of a 14-year-old Yorkshire terrier were submitted to histological examination. The dog showed 
chronic vomiting and a gastric mass was evident in abdominal sonography. During endoscopy and laparoscopy at the Small Animal Clinic, LMU Munich, a biopsy of a $3 \times 3 \times 4 \mathrm{~cm}$ mass located at the gastric cardia, and biopsy samples of liver, pancreas and lymph nodes were obtained and fixed in 10\% phosphate-buffered formalin. Biopsy samples were embedded in paraffin wax and routinely processed for light microscopy. Immunohistochemistry was performed on paraffin wax sections. Antigen retrieval was performed using proteinase $\mathrm{K}$ (synaptophysin, cytokeratin) or microwave pre-treatment with citrate buffer (pH 6.0) for 20 minutes (chromogranin A, Vimentin, S100). Primary antibodies used for immunohistochemistry included rabbit anti chromogranin A (1:500 in PBS pH 7.4, Dako Deutschland GmbH, Hamburg, Germany), rabbit anti neuron-specific enolase (1:500 in PBS, Biomol GmbH, Hamburg, Germany), rabbit anti synaptophysin (1:100 in PBS, Dako), mouse anti vimentin (1:300 in PBS, Dako), rabbit anti S100 (1:400 in PBS, Dako), and mouse anti cytokeratin (1:50 in PBS, Dako). A biotinylated secondary antibody was used to detect antibodies against chromogranin A, neuron-specific enolase, S100 and synaptophysin, using the avidin-biotin complex method (VECTOR Laboratories Ltd., Peterborough, United Kingdom), and a peroxidase conjugated antibody was used to detect antibodies against cytokeratin and vimentin (Dako); DAB served as chromogen. Samples of $1 \mathrm{~mm}^{3}$ were taken from paraffin blocks for transmission electron microscopy, dewaxed, contrasted in osmium tetroxide and embedded in Epon resin.

\section{Results}

Grossly, the gastric biopsy sample was irregularly shaped with a tan to brown cross-section. Histologically, the sample consisted of nests and cords of monomorphic round to oval cells, sometimes showing peripheral palisading. The tumour cells had eroded the mucosa and deeply invaded the submucosa and muscularis. Their cytoplasm was slightly granular and nuclei were round to oval. The mitotic index was low (2 - 10 per $2 \mathrm{~mm}^{2}$ ), and atypia were uncommon. The tumour cell nests and cords were separated by an abundant fibrovascular stroma with lymphocytic infiltrates sometimes forming follicles (Figures 1(a) and (b)). Vascular invasion into venous blood vessels was observed in some locations (Figure 1(c)). The lymph node biopsy contained numerous similar tumour cells, whereas the liver and pancreas samples showed no evidence of metastases (not shown).

Immunohistochemically, tumour cells of the stomach were positive for chromogranin A, synaptophysin, and cytokeratin. Few tumour cells were positive for S100 (sustentacular cells) and the tumour was negative for neuron-specific enolase and vimentin (Figures 2(a)-(f)),

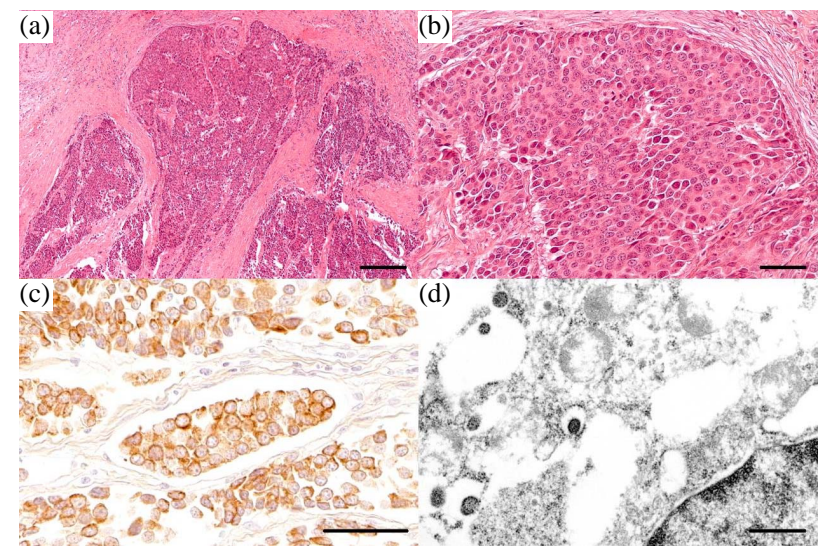

Figure 1. Histology of the gastric tumour. (a) Uniform tumour cells are arranged in nests and cords, separated by abundant fibrovascular stroma, multifocal mononuclear infiltrates may be seen in the surrounding connective tissue, H \& E stain, bar $200 \mu \mathrm{m}$; (b) Tumour cells arranged in nests with peripheral palisading, bar $50 \mu \mathrm{m}$; (c) Vascular invasion, anti-cytokeratin immunohistochemistry, bar 50 $\mu \mathrm{m}$; (d) Transmission electron microscopy showing electron dense secretory granules, bar $500 \mathrm{~nm}$.

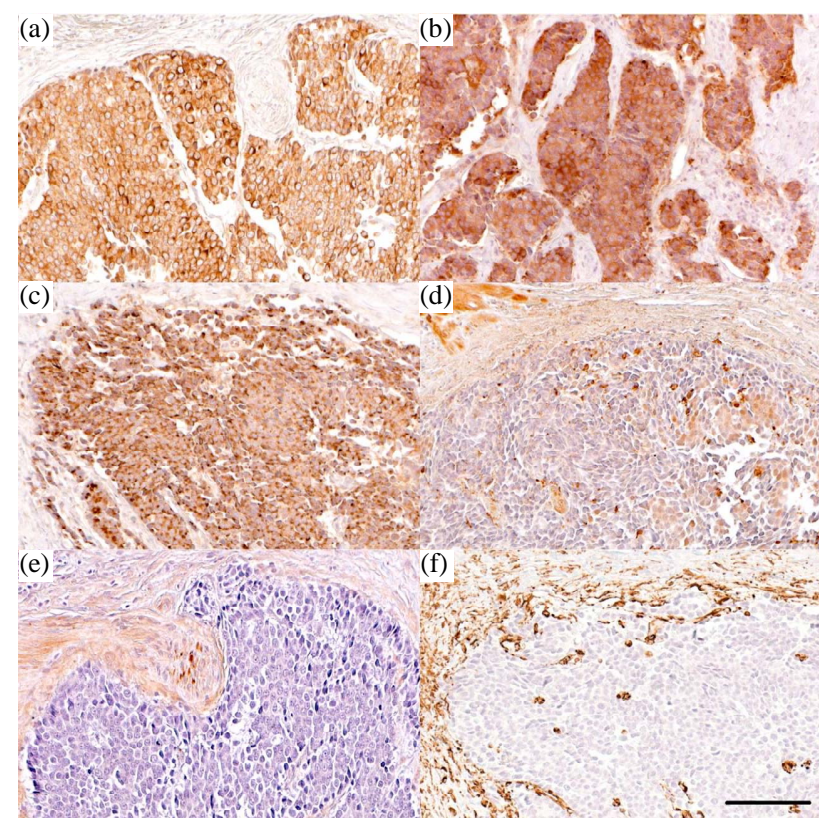

Figure 2. Immunohistochemistry of the gastric tumour. Immunohistochemical staining for (a) Cytokeratin; (b) Synaptophysin; (c) Chromogranin; (d) S100 protein; (e) Neuron-specific enolase; (f) Vimentin, bar $100 \mu \mathrm{m}$.

whereas the fibrovascular stroma was positive for vimentin. Electron microscopically, tumour cells exhibited few dense core secretory granules of $100-150 \mathrm{~nm}$ diameter, with a large halo and a well designated limiting membrane (Figure 1(d)). An intermediate grade gastric neuroendocrine carcinoma was diagnosed based on the location as well as histological, immunohistochemical and electron microscopic features of the tumour cells. 
The dog was euthanized two months after the diagnosis was made due to deterioration of the general condition and the poor prognosis. Unfortunately the body was not submitted to post mortem examination.

\section{Discussion}

Gastric tumours are rare in domestic animals and gastric NETs have only been reported in dogs to date [2]. According to the Veterinary WHO classification of tumours of the alimentary tract, NET of the stomach (also called carcinoid or neuroendocrine carcinoma, NEC) are defined as low-grade malignant tumours with neurosecretory granules that are identifiable light microscopically, immunohistochemically using special stains, or by electron microscopy $[2,6,7]$.

Well differentiated NETs cannot simply be classified by conventional histopathology with respect to their growth pattern and cellular features [1]. According to the human WHO classification, a gastric NEN larger than 2 $\mathrm{cm}$, invading the T. muscularis is considered a well differentiated low-grade NEC. Tumours up to $2 \mathrm{~cm}$, confined to the mucosa-submucosa with or without angioinvasion are classified as benign or low-grade NET and poorly differentiated NEC are high-grade malignant [8].

There are few case reports on gastric NETs in dogs. A gastric carcinoid was reported in a 13-year-old female whippet that was presented with a 10-day history of weakness and ataxia as well as was vomiting for 3 days. Post mortem examination showed a $3.5 \mathrm{~cm}$-diameter erosive transmural lesion on the antral mucosa of the stomach. Histologically, the tumour was described as a poorly differentiated NEC with high mitotic rate, multifocal necrosis and invasion of blood and lymphatic vessels. Metastases were observed in liver, spleen, kidney, lungs, lymph nodes and brain $[9,10]$. More recently, gastric carcinoids were reported in the Norwegian Lundehund, a breed often afflicted by chronic atrophic gastritis (CAG) and gastric tumours, including gastric carcinoma and NEC [11,12]. The Lundehund is thought to develop hyperplasia of gastric endocrine cells in response to decreased acid secretion and hypergastrinaemia, related to CAG [11]. Four of eight gastric tumour-bearing Lundehund exhibited gastric carcinoids, with metastases into regional lymph nodes. Three animals showed liver metastases, and in one dog, lung metastases were demonstrated. An association of chronic atrophic gastritis and gastric tumours is also described in humans that develop type 1 gastric NET as a result of increased gastrin production [1].

Male dogs seem predisposed to developing gastric tumours, and the dog in the present study as well as three of four affected Norwegian Lundehund were reported to be male $[6,11]$. In humans, male predominance is reported for sporadic (type 3) NET and undifferentiated gastric carcinoma (type 4 NET) [1].

Immunohistochemically, NET cells stain positive for chromogranin, synaptophysin, NCAM/CD56 and may be positive for a variety of peptide hormones (e.g. ACTH, calcitonin) and biogenic amines (e.g. serotonin) $[1,3,13]$. Likewise, the tumour in the present study was positive for chromogranin A, synaptophysin and cytokeratin, and the tumour of the female whippet, in contrast to the present case, was additionally positive for NSE [9]. In the Lundehund, gastric NET was confirmed by chromogranin A and synaptophysin immunohistochemistry [11]. Metastases were found in all reported cases. In the present study only the biopsy specimen of the lymph node showed metastases, however, due to the small size of pancreatic and liver biopsies that were taken during laparoscopy, we can not exclude distant metastases that may have been overlooked. The invasive growth and invasion of blood vessels however makes distant metastases likely and argues for malignant behaviour. The mitotic index of the gastric NET identified in the present report was fairly low (2 - 20 per $2 \mathrm{~mm}^{2}$ ), which is comparable to the mitotic index of gastric NET in the Lundehund but slightly lower than that of the female whippet $[9,11]$. According to the human and veterinary diagnostic criteria, the present case of a gastric NET would be designated as a well-differentiated intermediate-grade (G2) malignant NET/NEC.

\section{Acknowledgements}

We thank Elisabeth Kemper and Karin Stingl for excellent technical assistance

\section{REFERENCES}

[1] G. Klöppel, “Tumour Biology and Histopathology of Neuroendocrine Tumours," Best Practice \& Research: Clinical Endocrinology \& Metabolism, Vol. 21, No. 1, 2007, pp. 15-31. doi:10.1016/j.beem.2007.01.004

[2] K. W. Head, J. M. Cullen, R. R. Dubielzig, R. W. Else, W. Misdorp, A. K. Patnaik, S. Tateyama and I. van der Gaag, "Histological Classification of Gastric Tumors of Domestic Animals,” In: F. Y. Schulman, Ed., Histological Classification of Tumors of the Alimentary System of Domestic Animals, Armed Forces Institute of Pathology, Washington, 2003.

[3] M. Kiupel, C. Capen, M. Miller and R. Smedley, "Tumors of Dispersed Endocrine Cells,” Armed Forces Institute of Pathology, Washington, 2008.

[4] M. Schott, G. Klöppel, A. Raffel, A. Saleh, W. T. Knoefel and W. A. Scherbaum, "Neuroendocrine Neoplasms of the Gastrointestinal Tract,” Deutsches Ärzteblatt International, Vol. 108, No. 18, 2011, pp. 305-312.

[5] G. Rindi, R. Arnold, F. T. Bosmann, C. Capella, D. S. Klimstra, G. Klöppel, P. Komminoth and E. Solcia, "Nomenclature and Classification of Neuroendocrine Neo- 
plasms of the Digestive System,” In: F. T. Bosman, F. Carneiro, R. H. Hruban and N. D. Theise, Eds., WHO Classification of Tumours of the Digestive System, IARC, Lyon, 2010.

[6] C. C. Brown, D. C. Baker and I. K. Barker, "Neoplastic and Proliferative Lesions of the Stomach and Intestine," In: M. G. Maxie, Ed., Jubb, Kennedy and Palmer's Pathology of Domestic Animals, W. B. Saunders, Edinburgh, 2007, pp. 116-128.

[7] K. W. Head, R. W. Else and R. R. Dubielzig, "Tumors of the Alimentary Tract,” In: D. J. Meuten, Ed., Tumors in Domestic Animals, Iowa State Press, Iowa, 2002, pp. 401-481. doi:10.1002/9780470376928.ch8

[8] G. Kloppel, A. Perren and P. U. Heitz, "The Gastroenteropancreatic Neuroendocrine Cell System and Its Tumors: The WHO Classification," Annals of the New York Academy of Sciences, Vol. 1014, 2004, pp. 13-27. doi:10.1196/annals.1294.002

[9] T. M. Albers, J. Alroy, J. J. McDonnell and A. S. Moore, "A Poorly Differentiated Gastric Carcinoid in a Dog,"
Journal of Veterinary Diagnostic Investigation, Vol. 10, No. 1, 1998, pp. 116-118. doi: $10.1177 / 104063879801000126$

[10] D. G. Penninck, A. S. Moore and J. Gliatto, "Ultrasonography of Canine Gastric Epithelial Neoplasia,” Veterinary Radiology \& Ultrasound, Vol. 39, No. 4, 1998, pp. 342-348. doi:10.1111/j.1740-8261.1998.tb01618.x

[11] G. Qvigstad, O. Kolbjornsen, E. Skancke and H. L. Waldum, "Gastric Neuroendocrine Carcinoma Associated with Atrophic Gastritis in the Norwegian Lundehund," Journal of Comparative Pathology, Vol. 139, No. 4, 2008, pp. 194-201. doi:10.1016/j.jcpa.2008.07.001

[12] O. Kolbjornsen, C. M. Press and T. Landsverk, "Gastropathies in the Lundehund. I. Gastritis and Gastric Neoplasia Associated with Intestinal Lymphangiectasia,” APMIS, Vol. 102, No. 9, 1994, pp. 647-661.

[13] A. I. Baba and C. Câtoi, "Comparative Oncology," The Publishing House of the Romanian Academy, Bucharest, 2007. 